03

\title{
Электромагнитные волны в волноводе с периодически модулированным магнитодиэлектрическим заполнением
}

\author{
() Э.А. Геворкян \\ Российский экономический университет им. Г.В. Плеханова, \\ 117997 Москва, Россия \\ e-mail: gevor_mesi@mail.ru \\ Поступила в редакцию 31.01.2021 г. \\ В окончательной редакции 17.03.2021 г. \\ Принята к публикации 22.03.2021 г.
}

\begin{abstract}
Рассмотрено распространение электромагнитных волн в идеальном регулярном волноводе, магнитодиэлектрическое заполнение которого периодически модулировано в пространстве и во времени. Предполагается, что глубины модуляции - малые величины, и модуляция заполнения волновода не приводит к взаимодействию между различными волноводными модами. Получены волновые уравнения для поперечноэлектрического (ТЕ) и поперечно-магнитного (ТМ) полей в волноводе относительно продольных составляющих магнитного и электрического векторов соответственно. Они представляют дифференциальные уравнения в частных производных второго порядка с периодическими коэффициентами. Заменой переменных эти уравнения сводятся к обыкновенным дифференциальным уравнениям с периодическими коэффициентами типа Матье-Хилла. Найдены решения этих уравнений в первом приближении по малым глубинам модуляции в области „слабого“ взаимодействия между сигнальной волной и волной модуляции (условие Вульфа-Брэгга не выполняется). Полученные результаты показывают, что ТЕ- и ТМ-поля в волноводе в указанном выше приближении представляются в виде суммы трех пространственно-временных гармоник (нулевая и плюс и минус первые) со сложными амплитудами и частотами.
\end{abstract}

Ключевые слова: волновод, электромагнитная волна, модулированное заполнение, волновое уравнение, уравнение типа Матье-Хилла, глубины модуляции.

DOI: $10.21883 /$ OS.2021.07.51081.1865-21

\section{Введение}

В работах [1-8] были исследованы многие аспекты взаимодействия сигнальной электромагнитной волны с периодически модулированной диэлектрической средой в неограниченном пространстве и в регулярном волноводе произвольного поперечного сечения. Настоящая работа посвящена исследованию взаимодействия поперечноэлектрической (ТЕ) и поперечно-магнитной (ТМ) волн с периодически модулированной в пространстве и во времени магнитодиэлектрической средой в идеальном регулярном волноводе произвольного поперечного сечения. Подобное исследование представляет интерес не только с точки зрения развития теории электродинамики периодических сред, но и с точки зрения возможностей использования периодических сред в различных областях электроники СВЧ [2].

\section{Постановка задачи и ее решение}

Рассмотрим регулярный идеальный волновод произвольного поперечного сечения, ось которого совпадает с осью $O Z$ некоторой прямоугольной системы координат, а магнитодиэлектрическая среда в волноводе периодически модулирована в пространстве и во времени по закону

$$
\varepsilon(z, t)=\varepsilon^{0}\left[1+m_{\varepsilon} \cos k_{0}(z-u t)\right],
$$

$$
\mu(z, t)=\mu^{0}\left[1+m_{\mu} \cos k_{0}(z-u t)\right]
$$

где $k_{0}$ и $u-$ волновые числа и скорость волны модуляции, $m_{\varepsilon}$ и $m_{\mu}-$ малые глубины модуляции $\left(m_{\varepsilon} \ll 1, m_{\mu} \ll 1\right), \varepsilon^{0}$ и $\mu^{0}-$ диэлектрическая и магнитная проницаемости заполнения волновода в отсутствие модуляции. Пусть электромагнитная волна с частотой $\omega_{0}$ распространяется в подобном волноводе вдоль его оси в положительном направлении оси $O Z$.

Как известно $[4,6]$ ТЕ- и ТМ-поля в волноводе можно описывать с помощью продольных компонент магнитного и электрического векторов соответственно. Волновые уравнения для указанных компонент можно получить из системы уравнений Максвелла

$$
\begin{array}{r}
\operatorname{rot} \mathbf{H}=\frac{\partial \mathbf{D}}{\partial t}, \quad \operatorname{rot} \mathbf{E}=-\frac{\partial \mathbf{B}}{\partial t}, \quad \operatorname{div} \mathbf{D}=0, \\
\operatorname{div} \mathbf{B}=0, \quad \mathbf{D}=\varepsilon_{0} \varepsilon(z, t) \mathbf{E}, \quad \mathbf{B}=\mu_{0} \mu(z, t) \mathbf{H},
\end{array}
$$

где $\varepsilon(z, t)$ и $\mu(z, t)$ выражаются формулами (1) и (2), $\varepsilon_{0}=\left(36 \pi \cdot 10^{9}\right)^{-1} F / m$ - электрическая постоянная, $\mu_{0}=4 \pi \cdot 10^{-7} H / m-$ магнитная постоянная. Вычисления приводят к следующим дифференциальным уравнениям в частных производных второго порядка:

$$
\Delta_{\perp} \tilde{H}_{z}+\mu \frac{\partial}{\partial z}\left(\frac{1}{\mu} \frac{\partial \tilde{H}_{z}}{\partial z}\right)-\varepsilon_{0} \mu_{0} \mu \frac{\partial}{\partial t}\left(\varepsilon \frac{\partial \tilde{H}_{z}}{\partial t}\right)=0
$$




$$
\Delta_{\perp} \tilde{E}_{z}+\varepsilon \frac{\partial}{\partial z}\left(\frac{1}{\varepsilon} \frac{\partial \tilde{E}_{z}}{\partial z}\right)-\varepsilon_{0} \mu_{0} \varepsilon \frac{\partial}{\partial t}\left(\mu \frac{\partial \tilde{E}_{z}}{\partial t}\right)=0,
$$

где $\Delta_{\perp}=\partial^{2} / \partial x^{2}+\partial^{2} / \partial y^{2}-$ двумерный оператор Лапласа, а $\tilde{H}_{z}$ и $\tilde{E}_{z}$ определяются формулами

$$
\begin{aligned}
\tilde{H}_{z}(x, y, z, t) & =\mu(z, t) H_{z}(x, y, z, t), \\
\tilde{E}_{z}(x, y, z, t) & =\mu(z, t) E_{z}(x, y, z, t) .
\end{aligned}
$$

Если в уравнения (5) и (6) ввести новые переменные по формулам

$$
\xi=z-u t, \quad \eta=\frac{z}{u}-\frac{1}{u} \int_{0}^{\xi} \frac{d \xi}{1-\beta^{2} \varepsilon(\xi) \mu(\xi)},
$$

где $\beta^{2}=u^{2} \varepsilon_{0} \mu_{0}$, то они относительно переменных $\xi$ и $\eta$ преобразуются к виду

$\Delta_{\perp} \tilde{H}_{z}+\mu \frac{\partial}{\partial \xi}\left[\frac{1}{\mu}\left(1-\beta^{2} \varepsilon \mu\right) \frac{\partial \tilde{H}_{z}}{\partial \xi}\right]-\frac{\varepsilon_{0} \mu_{0} \varepsilon \mu}{1-\beta^{2} \varepsilon \mu} \frac{\partial^{2} \tilde{H}_{z}}{\partial \eta^{2}}=0$,

$\Delta_{\perp} \tilde{E}_{z}+\varepsilon \frac{\partial}{\partial \xi}\left[\frac{1}{\varepsilon}\left(1-\beta^{2} \varepsilon \mu\right) \frac{\partial \tilde{E}_{z}}{\partial \xi}\right]-\frac{\varepsilon_{0} \mu_{0} \varepsilon \mu}{1-\beta^{2} \varepsilon \mu} \frac{\partial^{2} \tilde{E}_{z}}{\partial \eta^{2}}=0$.

Решения уравнений (10) и (11) ищем в виде

$$
\begin{aligned}
& \tilde{H}_{z}(x, y, \xi, \eta)=\sum_{n=0}^{\infty} H_{n}(\xi) e^{i \eta \gamma} \widehat{\psi}_{n}(x, y), \\
& \tilde{E}_{z}(x, y, \xi, \eta)=\sum_{n=0}^{\infty} E_{n}(\xi) e^{i \eta \gamma} \psi_{n}(x, y),
\end{aligned}
$$

где $\widehat{\psi}_{n}(x, y)$ и $\psi_{n}(x, y)$ являются ортонормированными собственными функциями краевых задач Неймана (вторая) и Дирихле (первая) для поперечного сечения волновода и удовлетворяют уравнениям Гельмгольца с соответствующими граничными условиями:

$$
\begin{aligned}
& \Delta_{\perp} \widehat{\psi}_{n}(x, y)+\widehat{\lambda}_{n}^{2} \widehat{\psi}_{n}(x, y)=0,\left.\frac{\partial \widehat{\psi}_{n}(x, y)}{\partial \mathbf{n}}\right|_{\Sigma}=0, \\
& \Delta_{\perp} \psi_{n}(x, y)+\lambda_{n}^{2} \psi_{n}(x, y)=0,\left.\quad \psi_{n}(x, y)\right|_{\Sigma}=0 .
\end{aligned}
$$

Отметим, что в (14) и (15) $\hat{\lambda}_{n}$ и $\lambda_{n}-$ собственные значения краевых задач, соответствующих собственным функциям $\widehat{\psi}_{n}(x, y)$ и $\psi_{n}(x, y), \Sigma-$ контур поперечного сечения волновода, $\mathbf{n}-$ нормаль к $\Sigma$.

Любопытно заметить, что если решения волновых уравнений (5) и (6) искали бы в виде

$$
\begin{gathered}
\tilde{H}_{z}(x, y, z, t)=\sum_{n=0}^{\infty} H_{n}(z, t) \widehat{\psi}_{n}(x, y), \\
\tilde{E}_{z}(x, y, z, t)=\sum_{n=0}^{\infty} E_{n}(z, t) \psi_{n}(x, y),
\end{gathered}
$$

то из уравнений Максвелла (3) и (4) с учетом (14) и (15) для поперечных составляющих ТЕ- и ТМ-полей в волноводе получили бы следующие выражения:

$$
\begin{aligned}
\mathbf{H}_{\tau}^{\mathrm{TE}} & =\frac{1}{\mu(z, t)} \sum_{n=0}^{\infty} \widehat{\lambda}_{n}^{-2} \frac{\partial\left[\mu(z, t) H_{n}(z, t)\right]}{\partial z} \nabla \widehat{\psi}_{n}(x, y), \\
\mathbf{E}_{\tau}^{\mathrm{TE}} & =\mu_{0} \sum_{n=0}^{\infty} \widehat{\lambda}_{n}^{-2} \frac{\partial\left[\mu(z, t) H_{n}(z, t)\right]}{\partial z}\left[\mathbf{z}_{0} \nabla \widehat{\psi}_{n}(x, y)\right], \\
\mathbf{H}_{\tau}^{\mathrm{TE}} & =-\varepsilon_{0} \sum_{n=0}^{\infty} \lambda_{n}^{-2} \frac{\partial\left[\varepsilon(z, t) E_{n}(z, t)\right]}{\partial z}\left[\mathbf{z}_{0} \nabla \psi_{n}(x, y)\right], \\
\mathbf{E}_{\tau}^{\mathrm{TE}} & =\frac{1}{\varepsilon(z, t)} \sum_{n=0}^{\infty} \lambda_{n}^{-2} \frac{\partial\left[\varepsilon(z, t) E_{n}(z, t)\right]}{\partial z} \nabla \psi_{n}(x, y),
\end{aligned}
$$

где $\nabla=\mathbf{i}(\partial / \partial x)+\mathbf{j}(\partial / \partial y)$ - двумерный оператор набла, $\mathbf{z}_{0}-$ орт оси $O Z$ индекс $\tau$ указывает на поперечные составляющие.

Подставляя (12) и (13) в (10) и (11) и учитывая (14) и (15), после несложных преобразований получим следующие обыкновенные дифференциальные уравнения второго порядка относительно величин $H_{n}(\xi)$ и $E_{n}(\xi)$ :

$$
\begin{aligned}
& \mu \frac{d}{d \xi}\left[\frac{1}{\mu}\left(1-\beta^{2} \varepsilon \mu\right) \frac{d H_{n}(\xi)}{d \xi}\right]+\frac{\widehat{\chi}_{n}^{2}}{1-\beta^{2} \varepsilon \mu} H_{n}(\xi)=0, \\
& \varepsilon \frac{d}{d \xi}\left[\frac{1}{\varepsilon}\left(1-\beta^{2} \varepsilon \mu\right) \frac{d E_{n}(\xi)}{d \xi}\right]+\frac{\chi_{n}^{2}}{1-\beta^{2} \varepsilon \mu} E_{n}(\xi)=0
\end{aligned}
$$

где

$$
\begin{aligned}
\widehat{\chi}_{n}^{2} & =\varepsilon_{0} \mu_{0} \varepsilon \mu \gamma^{2}-\widehat{\lambda}_{n}^{2}\left(1-\beta^{2} \varepsilon \mu\right), \\
\chi_{n}^{2} & =\varepsilon_{0} \mu_{0} \varepsilon \mu \gamma^{2}-\lambda_{n}^{2}\left(1-\beta^{2} \varepsilon \mu\right) .
\end{aligned}
$$

Если в уравнения (22) и (23) ввести новые переменные по формулам

$$
\begin{gathered}
\widehat{s}=\frac{k_{0}\left(1-\beta^{2}\right)}{2 \mu_{0}} \int_{0}^{\xi} \frac{\mu d \xi}{1-\beta^{2} \varepsilon \mu}, \\
s=\frac{k_{0}\left(1-\beta^{2}\right)}{2 \varepsilon_{0}} \int_{0}^{\xi} \frac{\varepsilon d \xi}{1-\beta^{2} \varepsilon \mu},
\end{gathered}
$$

и учитывая, что $\varepsilon$ и $\mu$ выражаются формулами (1) и (2), то (22) и (23) преобразуются к дифференциальным уравнениям с периодическими коэффициентами типа Матье-Хилла. В первом приближении по малым глубинам модуляции они имеют вид

$$
\begin{gathered}
\frac{d^{2} H_{n}(\widehat{s})}{d \widehat{s}^{2}}+\left(\sum_{k=-1}^{1} \widehat{\theta}_{k}^{n} e^{2 i k \widehat{s}}\right) H_{n}(\widehat{s})=0, \\
\frac{d^{2} E_{n}(s)}{d s^{2}}+\left(\sum_{k=-1}^{1} \theta_{k}^{n} e^{2 i k s}\right) E_{n}(s)=0,
\end{gathered}
$$


где

$$
\begin{gathered}
\widehat{\theta}_{0}^{n}=\frac{4}{k_{0}^{2}\left(1-\beta^{2}\right)^{2}}\left(\widehat{\chi}_{0}^{n}\right)^{2}, \quad \theta_{0}^{n}=\frac{4}{k_{0}^{2}\left(1-\beta^{2}\right)^{2}}\left(\chi_{0}^{2}\right)^{2}, \\
\widehat{\theta}_{ \pm 1}^{n}=\frac{2}{k_{0}^{2}\left(1-\beta^{2}\right)^{2}}\left\{\left[\frac{\gamma^{2}}{u^{2}}-\left(\bar{\chi}_{0}^{n}\right)^{2}\right] l-2\left(\bar{\chi}_{0}^{n}\right)^{2} m_{\mu}\right\}, \\
\theta_{ \pm 1}^{n}=\frac{2}{k_{0}^{2}\left(1-\beta^{2}\right)^{2}}\left\{\left[\frac{\gamma^{2}}{u^{2}}-\left(\chi_{0}^{n}\right)^{2}\right] l-2\left(\chi_{0}^{n}\right)^{2} m_{\varepsilon}\right\}, \\
\left(\bar{\chi}_{0}^{n}\right)^{2}=\gamma^{2} \varepsilon^{0} \mu^{0} \varepsilon_{0} \mu_{0}-\widehat{\lambda}_{n}^{2}\left(1-\beta^{2}\right), \\
\left(\chi_{0}^{n}\right)^{2}=\gamma^{2} \varepsilon^{0} \mu^{0} \varepsilon_{0} \mu_{0}-\lambda_{n}^{2}\left(1-\beta^{2}\right),
\end{gathered}
$$

а величина $l=\left[\left(m_{e}+m_{\mu}\right) \beta^{2} /\left(1-\beta^{2}\right)\right] \ll 1$ так как $u \leq(0,8) / \sqrt{\varepsilon_{0} \mu_{0} \varepsilon^{0} \mu^{0}}$. Решения уравнений (27) и (28) ищем в виде

$$
\begin{gathered}
H_{n}(\widehat{s})=e^{i \widehat{\mu}_{n} \widehat{s}} \sum_{k=-1}^{1} \widehat{c}_{k}^{n} e^{2 i k \widehat{s}}, \\
E_{n}(s)=e^{i \mu_{n} s} \sum_{k=-1}^{1} c_{k}^{n} e^{2 i k s},
\end{gathered}
$$

где характеристические числа $\widehat{\mu}_{n}, \mu_{n}$ и коэффициенты $\widehat{c}_{k}^{n}, c_{k}^{n}-$ пока неизвестные величины. Для их определения потребуем, чтобы (33) и (34) удовлетворяли уравнениям (27) и (28). Это приводит к дисперсионным уравнениям задачи для определения $\widehat{\mu}_{n}, \mu_{n}$ и к системе алгебраических уравнений для определения $\widehat{c}_{k}^{n}, c_{k}^{n}$.

Отметим, что далее нас будет интересовать частотная область слабого взаимодействия между сигнальной волной и волной модуляции заполнения волновода, когда величины $\widehat{\theta}_{0}^{n}$ и $\theta_{0}^{n}$ не очень близки к единице (не выполняется условие Вульфа-Брэгга первого порядка и не происходит усиление отраженных от периодических неоднородностей заполнения волновода волн при их интерференции). В этой области из дисперсионных уравнений

$$
\begin{gathered}
\widehat{\mu}_{n}^{2}=\widehat{\theta}_{0}^{n}+\frac{\left(\hat{\theta}_{1}^{n}\right)^{2}}{\left(\widehat{\mu}_{n}-2\right)^{2}-\widehat{\theta}_{0}^{n}}+\frac{\left(\widehat{\theta}_{1}^{n}\right)^{2}}{\left(\widehat{\mu}_{n}-2\right)^{2}-\widehat{\theta}_{0}^{n}}, \\
\mu_{n}^{2}=\theta_{0}^{n}+\frac{\left(\theta_{1}^{n}\right)^{2}}{\left(\mu_{n}-2\right)^{2}-\theta_{0}^{n}}+\frac{\left(\theta_{1}^{n}\right)^{2}}{\left(\mu_{n}-2\right)^{2}-\theta_{0}^{n}},
\end{gathered}
$$

для характеристических чисел в первом приближении по малым глубинам модуляции получим

$$
\widehat{\mu}_{n}^{2}=\widehat{\theta}_{0}^{n}, \quad \mu_{n}^{2}=\theta_{0}^{n}
$$

Выражения для коэффициентов $\widehat{c}_{k}^{n}$ и $c_{k}^{n}$ в первом приближении по малым глубинам модуляции получаются из вышеуказанной системы алгебраических уравнений и имеют вид

$$
\widehat{c}_{ \pm 1}^{n} \simeq \frac{\widehat{\theta}_{1}^{n} \widehat{c}_{0}^{n}}{\left(\sqrt{\hat{\theta}_{0}^{n}} \pm 2\right)^{2}-\widehat{\theta}_{0}^{n}}, \quad c_{ \pm 1}^{n} \simeq \frac{\theta_{1}^{n} c_{0}^{n}}{\left(\sqrt{\theta_{0}^{n}} \pm 2\right)^{2}-\theta_{0}^{n}},
$$

где $\widehat{c}_{0}^{n}$ и $c_{0}^{n}$ можно определить из условий нормировки.

Если теперь подставить (33) и (34) в (12) и (13), перейти к переменным $z, t$ и одновременно пользоваться известным соотношением [9]

$$
e^{i \Delta \sin \varphi}=\sum_{k=-1}^{1} J_{h}(\Delta) e^{i h \varphi},
$$

где $\Delta-$ малый параметр, $J_{h}(\Delta)-$ функция Бесселя порядка $h$, то из (7) и (8) для продольных составляющих $H_{z}$ и $E_{z}$ TЕ- и ТМ-полей в волноводе с периодически модулированным магнитодиэлектрическим заполнением в области слабого взаимодействия между сигнальной волной и волной модуляции получим следующие выражения:

$$
\begin{aligned}
& H_{z}=\frac{1}{\mu^{0}} \sum_{n=0}^{\infty} \widehat{\psi}_{n}(x, y) e^{i\left(\hat{p}_{0}^{n} z-\omega_{0} t\right)} \widehat{c}_{0}^{n} \sum_{k=-1}^{1} \widehat{V}_{k}^{n} e^{i k k_{0}(z-u t),} \\
& E_{z}=\frac{1}{\varepsilon^{0}} \sum_{n=0}^{\infty} \psi_{n}(x, y) e^{i\left(p_{0}^{n} z-\omega_{0} t\right)} c_{0}^{n} \sum_{k=-1}^{1} V_{k}^{n} e^{i k k_{0}(z-u t),}
\end{aligned}
$$

где

$$
\begin{gathered}
\widehat{V}_{k}^{n}=\left(k \frac{\widehat{\Delta}_{0}^{n}}{2}+\frac{\widehat{c}_{k}^{n}}{\widehat{c}_{0}^{n}}-\frac{m_{\mu}}{2}\right)^{|k|}, \\
V_{k}^{n}=\left(k \frac{\Delta_{0}^{n}}{2}+\frac{c_{k}^{n}}{c_{0}^{n}}-\frac{m_{\varepsilon}}{2}\right)^{|k|}, \\
\widehat{\Delta_{0}^{n}}=\frac{\sqrt{\hat{\theta}_{0}^{n}}}{2} m_{\mu}+\frac{\omega_{0}}{k_{0} u} l, \Delta_{0}^{n}=\frac{\sqrt{\theta_{0}^{n}}}{2} m_{\varepsilon}+\frac{\omega_{0}}{k_{0} u} l, \\
\widehat{\theta}_{ \pm 1}^{n}=\frac{2\left[\left(\widehat{\chi}_{0}^{n}\right)^{2}+\widehat{\lambda}_{n}^{2}\right]}{k_{0}^{2} \beta^{2}\left(1-\beta^{2}\right)} l-\frac{4\left(\widehat{\chi}_{0}^{n}\right)^{2}}{k_{0}^{2}\left(1-\beta^{2}\right)^{2}} m_{\mu}, \\
\theta_{ \pm 1}^{n}=\frac{2\left[\left(\chi_{0}^{n}\right)^{2}+\lambda_{n}^{2}\right]}{k_{0}^{2} \beta^{2}\left(1-\beta^{2}\right)} l-\frac{4\left(\chi_{0}^{n}\right)^{2}}{k_{0}^{2}\left(1-\beta^{2}\right)^{2}} m_{\varepsilon}, \\
\left(\widehat{\chi}_{0}^{n}\right)^{2}=\left(\widehat{p}_{0}^{n} u-\omega_{0}\right)^{2} \varepsilon_{0} \mu_{0} \varepsilon^{0} \mu^{0}-\widehat{\lambda}_{n}^{2}\left(1-\beta^{2}\right), \\
\left(\chi_{0}^{n}\right)^{2}=\left(p_{0}^{n} u-\omega_{0}\right)^{2} \varepsilon_{0} \mu_{0} \varepsilon^{0} \mu^{0}-\lambda_{n}^{2}\left(1-\beta^{2}\right),
\end{gathered}
$$

$\left(\widehat{p}_{0}^{n}\right)^{2}=\omega_{0}^{2} \varepsilon_{0} \mu_{0} \varepsilon^{0} \mu^{0}-\widehat{\lambda}_{n}^{2}, \quad\left(p_{0}^{n}\right)^{2}=\omega_{0}^{2} \varepsilon_{0} \mu_{0} \varepsilon^{0} \mu^{0}-\lambda_{n}^{2}$. 


\section{Заключение}

Результаты, полученные в настоящей работе, показывают, что ТЕ- и ТМ-поля в волноводе с периодически модулированным в пространстве и во времени магнитодиэлектрическим заполнением в частотной области слабого взаимодействия сигнальной волны с волной модуляции, представляют сумму пространственновременны́х гармоник с различными сложными по характеру амплитудами и частотами. Показано, что амплитуда на основной (нулевой) гармонике не зависит от малых глубин модуляции, а на боковых (плюс и минус первых) гармониках амплитуды зависят от глубин модуляции в первой степени. Отметим также, что, основываясь на полученных в настоящей работе результатах, можно исследовать особенности распространения сигнальной волны в волноводе с периодически модулированным заполнением в области „сильного“ взаимодействия сигнальной волны с волной модуляции заполнения (выполняется условие Вульфа-Брэгга первого порядка). Развитый в настоящей работе аналитический метод позволит также решить задачу распространения электромагнитных волн в волноводе с многопериодически модулированным в пространстве и во времени магнитодиэлектрическим заполнением.

\section{Конфликт интересов}

Автор заявляет, что у него нет конфликта интересов.

\section{Список литературы}

[1] Барсуков К.А. // Радиотехника и электроника. 1964. Т. 9. № 7. C. 1173.

[2] Elachi Ch. // Proc. IEEE. 1976. V. 64. N 12. P. 1666.

[3] Gevorkyan E.A. // Physica A. 1977. V. 241. Р. 236.

[4] Барсуков К.А., Геворкян Э.А. // Радиотехника и электроника. 1983. Т. 28. В. 2. С. 237; Barsukov K.A., Gevorkyan E.A. // Radio Engineering and Electronic Physics. 1983. V. 28. N 2. P. 19.

[5] Столяров С.Н., Карпов С.Ю. // УФН. 1993. Т. 163. № 1. C. 63.

[6] Геворкян Э.А. // ЖТФ. 2006. Т. 76. В. 5. С. 134; Gevorkyan E.A. // Technical Physics. 2006. V. 51. N 5. P. 666.

[7] Gevorkyan E.A. // Acta Physica Polonica A. 2019. V. 135. N 4. P. 650. doi 10.12693/APhys PolA.135.650

[8] Gevorkyan E.A. // Proc. ICEAA-IEEE APWC-2019. 9-13 September 2019. Spain, Granada. IEEE Xplore Digital Library. 2019. P. 0031. doi 10.1109/ICEAA.2019.8879264

[9] Тихонов А.Н., Самарский А.А. Уравнения математической физики. М.: МГУ, 2004. 800 с. 